\title{
On differentiable compactifications of the hyperbolic plane and algebraic actions of $\mathrm{SL}_{2}(\mathbb{R})$ on surfaces
}

\author{
Benoît Kloeckner
}

Published online: 22 June 2007

(C) Springer Science+Business Media B.V. 2007

\section{Erratum to: Geom Dedicata \\ DOI: 10.1007/s10711-005-9020-5}

\begin{abstract}
Real-analytic actions of $\operatorname{SL}(2 ; \mathrm{R})$ on surfaces have been classified, up to analytic change of coordinates. In particular it is known that there exists countably many analytic equivariant compactification of the isometric action on the hyperbolic plane. In this paper we study the algebraicity of these actions. We get a classification of the algebraic actions of SL $(2 ; \mathrm{R})$ on surfaces. In particular, we classify the algebraic equivariant compactifications of the hyperbolic plane.
\end{abstract}

Keywords Differentiable compactification $\cdot$ Hyperbolic plane $\cdot \operatorname{SL}(2 ; \mathrm{R})$

Mathematics Subject Classification (2000) 57M60 (53C35)

\section{Introduction}

An important role played by $\mathrm{SL}_{2}(\mathbb{R})$ is its isometric action on the hyperbolic plane $\mathbb{H}^{2}$, which can be described as the homogeneous space $\mathrm{SL}_{2}(\mathbb{R}) / \mathrm{SO}_{2}(\mathbb{R})$, denoted by $\mathcal{E}$. This action is real analytic and is, up to analytic change of coordinates, the only real analytic transitive action of $\mathrm{SL}_{2}(\mathbb{R})$ on the open disk.

This is an Erratum to article "On Differentiable Compactifications of the Hyperbolic Plane and Algebraic Actions of SL2 $(\mathbb{R})$ on Surfaces" by Benoît Kloeckner which appeared in Volume 117 of Geometriae Dedicata (DOI: 10.1007/s10711-005-9020-5). The published article contained several errors, hence it is reprinted here.

The online version of the original article can be found under doi: 10.1007/s10711-005-9020-5.

B. Kloeckner $(\varangle)$

UMPA, ÉNS Lyon 46, allée d'Italie, 69364 Lyon cedex 07, France

e-mail: bkloeckn@umpa.ens-lyon.fr 
The notion of asymptotic geodesics is a means of understanding the behavior at infinity of this action, that is to say of giving a natural topological equivariant compactification of this action to an action on the closed disk.

One can ask whether there is a differentiable equivariant compactification of this action into the closed disk. The answer is positive, and there are two well-known ways to achieve such a compactification.

The restriction to $\mathrm{SL}_{2}(\mathbb{R})$ of the natural action of $\mathrm{SL}_{2}(\mathbb{C})$ on the Riemann sphere $\overline{\mathbb{C}}$ has three orbits: two open hemispheres and between them a great circle. Considering the union of one open orbit and the circle, one gets an analytic equivariant compactification of $\mathcal{E}$. We call it the conformal action. It corresponds to the continuous prolongation to the closed unit disk of the $\mathrm{SL}_{2}(\mathbb{R})$ action on Poincaré's disk.

One can also realize the hyperbolic plane by taking a lorentzian scalar product $Q$ on $\mathbb{R}^{3}: \mathrm{SL}_{2}(\mathbb{R})$ acts isometrically on $\left(\mathbb{R}^{3}, Q\right)$, and when one projectivizes $\mathbb{R}^{3}$ it gives an analytic action of $\mathrm{SL}_{2}(\mathbb{R})$ on $\mathbb{R} \mathbb{P}^{2}$ with three orbits: an open disk (which is the hyperbolic plane), an open Möbius strip and between them a circle. By taking the action of $\mathrm{SL}_{2}(\mathbb{R})$ on the union of the disk and the circle we get another analytic equivariant compactification of $\mathcal{E}$, called the projective action. It corresponds to the continuous prolongation to the closed unit disk of the $\mathrm{SL}_{2}(\mathbb{R})$ action on Klein's disk.

These two compactifications are topologically conjugate: they coincide with the Hadamard compactification. However it is easy to check the following already known but striking fact :

Proposition 1.1 The conformal and projective actions are not $\mathcal{C}^{1}$ conjugate, and in particular not $\mathcal{C}^{\omega}$ conjugate.

Proof If we choose a point $x$ of the disk boundary and consider in Poincaré's model the closure of the geodesics which have $x$ as an endpoint, we see that all of them are tangent, hence the differential in $x$ of the conformal action of the parabolic elements of $\mathrm{SL}_{2}(\mathbb{R})$ which fix $x$ have a common proper direction transversal to the boundary.

If we now consider the same geodesics in Klein's model, we see that no two of them are tangent and for each line of the tangent space in $x$, there is a closure of a geodesic tangent to it. Hence, the differential in $x$ of the projective action of a parabolic element of $\mathrm{SL}_{2}(\mathbb{R})$ which fixes $x$ has no proper direction transversal to the boundary.

One can ask whether these two compactifications are the only ones. The answer, stated in a different way, was given by Schneider [2] and Stowe [4]: there exists a countable family of non-equivalent analytic compactifications of $\mathcal{E}$, which can be described in terms of infinitesimal generators (see Sect. 4.2.1). These authors also describe all the analytic actions of $\mathrm{SL}_{2}(\mathbb{R})$ on compact surfaces with or without boundary and on $\mathbb{R}^{2}$.

However, these new actions seem less natural than the two compactifications we discussed before, which have well-known explicit integral models. Both of these models come in a certain sense from the projectivization of a linear representation; they will be called algebraic in the following sense:

Definition 1.2 Let $k$ be a positive integer, possibly $\infty$ or $\omega$. A $\mathcal{C}^{k}$ action $\alpha$ of $\mathrm{SL}_{2}(\mathbb{R})$ on a manifold $M$, possibly with boundary, is said to be $\mathcal{C}^{k}$-algebraic if there exists a continuous linear representation $\rho$ of $\mathrm{SL}_{2}(\mathbb{R})$ on a real finite-dimensional vectorial space $V$ and an $\mathcal{C}^{k}$ embedding $\Phi: M \longrightarrow \mathbb{P}(V)$ equivariant with respect to $\alpha$ and the projective representation $\mathbb{P}(\rho)$ induced by $\rho$ on $\mathbb{P}(V)$.

The pair $(\rho, \Phi)$ is called a $\mathcal{C}^{k}$ algebraic realization of $\alpha$.

黑 Springer 
It is obvious that the projective action is algebraic.

The Riemann sphere can be seen as a submanifold of the space of the 2-plans of $\mathbb{R}^{4}$ which, as a Grassmanian, can be embedded in a real projective space such that the conformal action of $\mathrm{SL}_{2}(\mathbb{R})$ extends to the projectivization of a linear representation. So the conformal action is algebraic too.

By studying the topology of all the algebraic continuous actions of $\mathrm{SL}_{2}(\mathbb{R})$ on surfaces and determining the regularity of the gluing of the orbits we prove (for a precise definition of "compactification" see Sect. 4.2.1):

Theorem 1.3 The conformal and projective actions are the only $\mathcal{C}^{\omega}$ compactifications of $\mathcal{E}$ which are algebraic.

This result is restated with our notations as Theorem 4.4. For the differentiable case, see Theorem 4.6.

With this material, we are also able to study all the analytic algebraic actions of $\mathrm{SL}_{2}(\mathbb{R})$ on surfaces and prove the following result.

Theorem 1.4 The analytic algebraic actions of $\mathrm{SL}_{2}(\mathbb{R})$ on compact surfaces (with or without boundary) that have an open orbit consist exactly of:

- the projective action (on $\left.\mathbb{R P}^{2}\right)$,

- the conformal action (on $\left.\mathbb{S}^{2}\right)$,

- the standard product action on $\mathbb{R P}^{1} \times \mathbb{R P}^{1}$,

- one action on the projective plane with an open dense orbit,

- a countable family of actions on the Klein bottle,

- a countable family of actions on the torus with two open cylindric orbits and two circular orbits,

- a countable family of actions on the torus with four open cylindric orbits and four circular orbits,

and of any subaction (i.e., union of orbits) of any one of these actions.

The proof goes through all the paper, and is summarized in Sect. 4.5

Note that the realizations of these actions as algebraic actions gives explicit global models for all of them.

\section{The topology of low dimensional algebraic orbits}

Our goal in this section is to describe the topology of all orbits of dimension less or equal to 2 which appear in the projectivization of a finite-dimensional linear representation of $\mathrm{SL}_{2}(\mathbb{R})$.

\subsection{Irreducible representations}

All the irreducible representations of $\mathrm{SL}_{2}(\mathbb{R})$ are known; for a proof of the following theorem, see [3].

We define a family of linear representations of $\mathrm{SL}_{2}(\mathbb{R})$. For each nonnegative integer $n, \rho_{n}: \mathrm{SL}_{2}(\mathbb{R}) \longrightarrow \mathbb{R}_{n}[X, Y]$, where $\mathbb{R}_{n}[X, Y]$ is the vector space of all homogeneous polynomials of degree $n$ in $X$ and $Y$, is given by

$$
\tilde{\rho}_{n}\left(\begin{array}{ll}
a & b \\
c & d
\end{array}\right) \cdot P(X, Y)=P(a X+c Y, b X+d Y) .
$$


Theorem 2.1 The $(n+1)$-dimensional representation $\rho_{n}$ is irreducible for any non-negative $n$ and any finite-dimensional irreducible representation of $\mathrm{SL}_{2}(\mathbb{R})$ is of this form.

\subsection{Irreducible case}

We start the study by the irreducible case.

The irreducible representation of dimension $1, \rho_{0}$, is trivial: its associated projective action has one single (fixed!) point.

The irreducible representation of dimension $2, \rho_{1}$, gives the usual action of $\mathrm{SL}_{2}(\mathbb{R})$ on $\mathbb{R} \mathbb{P}^{1}$, which is transitive.

The irreducible representation of dimension 3, $\rho_{2}$, gives the projective action on $\mathbb{R P}^{2}$, which has three orbits : one open disk, one circle and one Möbius strip. We can determine in which orbit lies the vector line given by a polynomial $P=a X^{2}+b X Y+$ $c Y^{2}$ (we denote such a line by $\left[a X^{2}+b X Y+c Y^{2}\right]$ ) just by computing the discriminant $\Delta=b^{2}-4 a c$ (which plays the role of the Lorentzian scalar product in the description of the projective action given in the Introduction). The open disk consists of the elements which are not factorizable over $\mathbb{R}$ (i.e. of negative discriminant). The Möbius strip consists of those which are factorizable with two distinct factors (i.e. of positive discriminant). The circle consists of those which are squares (i.e. of zero discriminant).

We denote by $\mathbb{H}^{+}$the upper half plane in $\mathbb{C}$ and by $\partial \mathbb{H}^{+}$its boundary (in Riemann's sphere $\overline{\mathbb{C}})$. We have a canonical identification between $\partial \mathbb{H}^{+}$and $\mathbb{R P}^{1}$.

It is important to notice that, since the map:

$$
\begin{aligned}
\mathbb{H}^{+} \sqcup \partial \mathbb{H}^{+} & \longrightarrow \mathbb{P}\left(\mathbb{R}_{3}[X, Y]\right) \\
z & \longmapsto[(z X+Y)(\bar{z} X+Y)]
\end{aligned}
$$

is not differentiable on the boundary, it is not an analytic parametrization of the closed disk (union of the open disk orbit and of the circular orbit) and there is no reason to think that the conformal and projective actions on the closed disk are equal up to analytic coordinate change (we already saw that they are not).

Now we generalize this method for all irreducible representations. Fix a positive integer $n$. An element of $\mathbb{P}\left(\mathbb{R}_{n}[X, Y]\right)$ factorizes into the following form:

$$
\left[\prod_{i=1}^{k}\left(t_{i} X+Y\right)^{\alpha_{i}} \prod_{j=1}^{l}\left(z_{j} X+Y\right)^{\beta_{j}}\left(\overline{z_{j}} X+Y\right)^{\beta_{j}}\right],
$$

where the $t_{i}$ 's are distinct elements of $\partial \mathbb{H}^{+}$, the $z_{j}$ 's are distinct elements of $\mathbb{H}^{+}$and $\sum \alpha_{i}+2 \sum \beta_{j}=n$.

Note that the $t_{i}$ 's are possibly infinite : for example $[\infty X+Y]$ denotes the projective element $[X]$.

The form (1) is efficient: since we have

$$
\left(\begin{array}{ll}
a & b \\
c & d
\end{array}\right) \cdot(z X+Y)=(c z+d)\left(\frac{a z+b}{c z+d} X+Y\right)
$$

where $z \in \overline{\mathbb{C}}$, the conformal action allows one to study all algebraic actions of $\mathrm{SL}_{2}(\mathbb{R})$ topologically.

We shall first determine which orbits are of dimension 2 or less. 
Lemma 2.2 The orbit of an element $P$ written under the form (1) is of dimension 2 or less if and only if: $k+2 l \leq 2$.

Proof We consider the different cases one by one. By 'isometry' we shall always mean 'orientation-preserving isometry'.

If $l=1$ and $k=1$, we can write $P=\left[(t X+Y)^{\alpha}(z X+Y)^{\beta}(\bar{z} X+Y)^{\beta}\right]$ and the stabilizer of $P$ is the set of the isometries of $\mathbb{H}^{+}$(with the hyperbolic metric) which fix the point $z$ and the point of the boundary $t$, and hence consist only of the identity Id. Thus the orbit of $P$ is of the same dimension as $\mathrm{SL}_{2}(\mathbb{R})$, i.e., 3 .

If $l \geq 1$ and $k \geq 1$, the same conclusion holds.

If $l \geq 2$, an element of the component of Id in the stabilizer of $P$ must fix at least two points of $\mathbb{H}^{+}$, hence the stabilizer is discrete and the orbit of $P$ is of dimension 3 .

If $k \geq 3$, an element of the component of Id of the stabilizer of $P$ must fix at least three points of the boundary $\partial \mathbb{H}^{+}$, hence the same conclusion holds.

If $l=0$ and $k=1$, the stabilizer of $P$ is the set of the isometries of $\mathbb{H}^{+}$which fix one given point (the only root of a representative polynomial for $P$ ) of the boundary, hence its dimension is 2 . Thus the orbit of $P$ is one-dimensional.

If $l=0$ and $k=2$, the stabilizer of $P$ is the set of the isometries of $\mathbb{H}^{+}$which fix two given points of the boundary, hence it is one-dimensional. Thus the dimension of the orbit of $P$ is 2 .

If $l=1$ and $k=0$ the stabilizer of $P$ is the set of the isometries of $\mathbb{H}^{+}$which fix one given point, hence it is one-dimensional. Thus the dimension of the orbit of $P$ is 2 .

We have three cases of low dimensional orbits, namely the elliptic case ( $l=1$ and $k=0)$, the parabolic case $(l=0$ and $k=1)$ and the hyperbolic case $(l=0$ and $k=2)$.

Proposition 2.3 The topology of an orbit of dimension 2 or less of the action $\mathbb{P}\left(\rho_{n}\right)$ (obtained by projectivizing $\rho_{n}$ ) is given by the factorized form (1) of any one of its elements $P$ in the following way:

(1) if $l=0$ and $k=1$ : the orbit of $P$ is a circle

$$
\left\{\left[(t X+Y)^{n}\right] ; t \in \partial \mathbb{H}^{+}\right\} .
$$

There is only one such orbit,

(2) if $l=0, k=2$ and $\alpha_{1}=\alpha_{2}$ : the orbit of $P$ is a Möbius strip

$$
\left\{\left[\left(t_{1} X+Y\right)^{\alpha}\left(t_{2} X+Y\right)^{\alpha}\right] ; t_{1} \neq t_{2} \in \partial \mathbb{H}^{+}\right\},
$$

where $t_{1}$ and $t_{2}$ play the same role. There is one such orbit if $n$ is even, none if $n$ is odd,

(3) if $l=0, k=2$ and $\alpha_{1} \neq \alpha_{2}$ : the orbit of $P$ is a cylinder

$$
\left\{\left[\left(t_{1} X+Y\right)^{\alpha_{1}}\left(t_{2} X+Y\right)^{\alpha_{2}}\right] ; t_{1} \neq t_{2} \in \partial \mathbb{H}^{+}\right\},
$$

where $t_{1}$ and $t_{2}$ play non symmetric roles (inverting them maps an element of the orbit to another). There are $(n-1) / 2$ such orbits if $n$ is odd, $(n-2) / 2$ if $n$ is even,

(4) if $l=1$ and $k=0$ : the orbit of $P$ is a disk

$$
\left\{\left[(z X+Y)^{\beta}(\bar{z} X+Y)^{\beta}\right] ; z \in \mathbb{H}^{+}\right\} .
$$

There is one such orbit if $n$ is even, none if $n$ is odd. 
Proof $\mathrm{As} \mathrm{SL}_{2}(\mathbb{R})$ is transitive on $\mathbb{H}^{+}$and doubly transitive on $\partial \mathbb{H}^{+}$, each set described here is an orbit. Thanks to Lemma 2.2 there is no other case than these four ones. The computation of the number of orbits is easy with the condition $\sum \alpha_{i}+2 \sum \beta_{j}=n$.

All we have to prove is that the topology of each of these sets is as claimed. The cases 1, 2, 4 can be deduced from the study of $\rho_{2}$ since the map

$$
\begin{aligned}
\mathbb{P}\left(\mathbb{R}_{m}[X, Y]\right) & \longrightarrow \mathbb{P}\left(\mathbb{R}_{\alpha m}[X, Y]\right) \\
{[P] } & \longmapsto\left[P^{\alpha}\right]
\end{aligned}
$$

is a homeomorphism on its image.

The case 3 reduces to the elementary fact that

$$
\left\{(x, y) \in \mathbb{S}^{1} \times \mathbb{S}^{1} ; x \neq y\right\}
$$

is a cylinder.

\subsection{Notations for the reducible case}

We shall now consider the reducible representations of $\mathrm{SL}_{2}(\mathbb{R})$. Since it is a semi-simple Lie group, its finite-dimensional representations are sums of irreducible representations. If we consider a representation $\rho$, we can write:

$$
\rho=\rho_{n_{1}} \oplus \rho_{n_{2}} \oplus \cdots \rho_{n_{p}}
$$

for some $n_{1}, \ldots, n_{p}$.

We denote by $V$ the vector space of $\rho$; we get

$$
V=\mathbb{R}_{n_{1}}[X, Y] \oplus \mathbb{R}_{n_{2}}[X, Y] \oplus \cdots \oplus \mathbb{R}_{n_{p}}[X, Y] .
$$

Up to a permutation, we can assume that $n_{1} \geq n_{2} \geq \cdots \geq n_{p}$.

Moreover, as we want to consider together all the copies of a given irreducible representation which appears in $\rho$ we set $I_{1}=\llbracket i_{1}, i_{2}-1 \rrbracket, I_{2}=\llbracket i_{2}, i_{3}-1 \rrbracket, \ldots$, $I_{r}=\llbracket i_{r}, i_{r+1}-1 \rrbracket\left(\right.$ where $i_{1}=1$ and $i_{r+1}-1=p$ ) the integer intervals such that:

$$
\underbrace{n_{1}=\cdots=n_{i_{2}-1}}_{I_{1}}>\underbrace{n_{i_{2}}=\cdots=n_{i_{3}-1}}_{I_{2}}>\cdots>\underbrace{n_{i_{r}}=\cdots=n_{p}}_{I_{r}} .
$$

We say that $I_{S}$ is even, respectively odd if $n_{i_{s}}$ is even, respectively odd.

We write an element $x$ of $\mathbb{P}(V)$ under the factorized form:

$$
x=\left[u_{q} \prod_{i=1}^{k_{q}}\left(t_{q}^{i} X+Y\right)^{\alpha_{q}^{i}} \prod_{j=1}^{l_{q}}\left(z_{q}^{j} X+Y\right)^{\beta_{q}^{j}}\left(z_{q}^{j} X+Y\right)^{\beta_{q}^{j}}\right]_{1 \leq q \leq p},
$$

where the $u_{q}$ 's are real numbers and for each $q$ : $\sum \alpha_{q}^{i}+2 \sum \beta_{q}^{j}=n_{q}$.

We call support of $x$ (or of the projective element $\left[u_{1}, \ldots, u_{p}\right]$ ) and denote by $I(x)$ the set of all the intervals $I_{S}$ such that there is at least one index $i \in I_{s}, u_{i} \neq 0$. We write $q \in I(x)$ instead of $q \in \bigcup_{I \in I(x)} I$.

We say that a support is even, respectively odd if all of its elements are even, respectively odd.

We denote by $I_{+}(x)$ the element of the support of $x$ which carries the greatest dimension (i.e., the lowest indices), $I_{-}(x)$ the one which carries the lowest dimension. 
We denote by $q_{+}(x)$ (respectively $\left.q_{-}(x)\right)$ the smallest (respectively the greatest) index $q$ such that $u_{q} \neq 0$. We have $q_{+}(x) \in I_{+}(x)$ and $q_{-}(x) \in I_{-}(x)$.

When there is no ambiguity, we write $I_{+}, I_{-}, q_{+}$and $q_{-}$instead of $I_{+}(x), I_{-}(x)$, $q_{+}(x)$ and $q_{-}(x)$.

We denote by $k(x)$ (or $k$ ) the number of different $t_{q}^{i}$ 's of $\partial \mathbb{H}^{+}$which arise in the factorized form (2) of $x$, and $l(x)$ (or $l$ ) the number of different $z_{q}^{j}$,s of $\mathbb{H}^{+}$.

With these notations we can now generalise the results of the previous section to reducible representations.

Lemma 2.4 Let $x$ be a element of the projective space $\mathbb{P}(V)$ whose orbit is of dimension 2 or less. Then $k(x)+2 l(x) \leq 2$.

Proof An element of the identity component of the stabilizer of $x$ is an isometry of $\mathbb{H}^{+}$stabilizing $l(x)$ points and $k(x)$ points of the boundary, so we can conclude using the discussion in the proof of Lemma 2.2.

Until the end of the paper, we shall assume there is at least one index $i$ such that $n_{i}>1$ (otherwise the action of $\mathrm{SL}_{2}(\mathbb{R})$ is trivial).

\subsection{Reducible elliptic case}

We assume here that $k=0$ and $l=1$, that is to say we consider the orbit of an element

$$
x=\left[u_{q}(z X+Y)^{\frac{n_{q}}{2}}(\bar{z} X+Y)^{\frac{n_{q}}{2}}\right]_{1 \leq q \leq p}
$$

which must be of even support.

Lemma 2.5 The orbit of an elliptic element is homeomorphic to a disk.

Proof Composing with an element of $\mathrm{SL}_{2}(\mathbb{R})$, we can assume $z=\imath$. Thus the elements of the stabilizer of $x$ are exactly the matrices $\left(\begin{array}{cc}a & b \\ -b & a\end{array}\right)$ where $a^{2}+b^{2}=1$.

Hence we can parametrize the orbit of $x$ by $z \in \mathbb{H}^{+}$.

\subsection{Reducible parabolic case}

Now we shall assume $k=1$ and $l=0$ and consider an element $x=\left[u_{q} Y^{n_{q}}\right]$ (after possible composition with an element of $\mathrm{SL}_{2}(\mathbb{R})$ ).

Lemma 2.6 The orbit of a parabolic element with support reduced to a single element is homeomorphic to a circle.

The orbit of a parabolic element with support containing at least two elements is homeomorphic to a cylinder.

Proof If $A=\left(\begin{array}{ll}a & b \\ c & d\end{array}\right)$ stabilizes $x$, it stabilizes 0 when acting projectively on $\mathbb{R P}^{1}$ thus $b=0\left(\right.$ and $\left.d=a^{-1}\right)$. 
Moreover we have

$$
\left(\begin{array}{cc}
a & 0 \\
c & a^{-1}
\end{array}\right) \cdot x=\left[u_{q} a^{-n_{q}} Y^{n_{q}}\right]_{q} .
$$

If the support of $x$ consists of one single interval $I_{S}$ the condition $b=0$ is sufficient for $A$ to stabilize $x$. If $d \neq 0$,

$$
\left(\begin{array}{ll}
a & b \\
c & d
\end{array}\right) \cdot x=\left[u_{q}\left(\frac{b}{d} X+Y\right)^{n_{q}}\right]_{q \in I_{s}}
$$

else

$$
\left(\begin{array}{ll}
a & b \\
c & 0
\end{array}\right) \cdot x=\left[u_{q} X^{n_{q}}\right]_{q \in I_{s}}
$$

Hence the orbit of $x$ is homeomorphic to $\mathbb{R P}^{1}$.

If the support of $x$ consists of at least two intervals the stabilizer of $x$ consists of the matrices of the form $A=\left(\begin{array}{ll}1 & 0 \\ c & 1\end{array}\right)$ hence the orbit is of dimension 2 .

If $d \neq 0$,

$$
\left(\begin{array}{ll}
a & b \\
c & d
\end{array}\right) \cdot x=\left[u_{q} d^{n_{q}}\left(\frac{b}{d} X+Y\right)^{n_{q}}\right]_{q}
$$

else

$$
\left(\begin{array}{ll}
a & b \\
c & 0
\end{array}\right) \cdot x=\left[u_{q} b^{n_{q}} X^{n_{q}}\right]_{q}
$$

hence a point of the orbit of $x$ is determined by $\frac{b}{d} \in \mathbb{R P}^{1}$ and a real non-zero parameter, $b$ or $d$. The case $d \neq 0$ gives a pair of disjoint copies of $\mathbb{R} \times \mathbb{R}^{*}$ which are glued along $d=0$ into a cylinder. If the support of $x$ is neither even nor odd this cylinder is naturally homeomorphic to the orbit of $x$, otherwise $\left(\begin{array}{cc}-a & -b \\ c & -d\end{array}\right) \cdot x=\left(\begin{array}{ll}a & b \\ c & d\end{array}\right) \cdot x$ and it is naturally a 2-folded covering of the orbit of $x$, which is a cylinder too.

\subsection{Reducible hyperbolic case}

We shall assume $k=2$ and $l=0$ and consider an element

$$
x=\left[u_{q} X^{\alpha_{q}} Y^{n_{q}-\alpha_{q}}\right]_{q}
$$

(note that we define $\alpha_{q}$ only when $u_{q} \neq 0$ ).

Lemma 2.7 With the notations of this section, a hyperbolic element has a 2 dimensional orbit if and only if $2 \alpha_{q}-n_{q}$ is constant, noted $\delta$. When this condition is satisfied, the orbit is a Möbius strip if $\delta=0$ and $\alpha_{q_{+}}-\alpha_{q}$ is even for each $q$, a cylinder otherwise.

Proof A stabilizing element of $x$ must stabilize 0 and $\infty$ in $\overline{\mathbb{C}}$ hence can be written $\left(\begin{array}{cc}a & 0 \\ 0 & a^{-1}\end{array}\right)$. As

$$
\left(\begin{array}{cc}
a & 0 \\
0 & a^{-1}
\end{array}\right) \cdot x=\left[u_{q} a^{2 \alpha_{q}-n_{q}} X^{\alpha_{q}} Y^{n_{q}-\alpha_{q}}\right]
$$


we see that if there are $q_{1}, q_{2}$ such that $2 \alpha_{q_{1}}-n_{q_{1}} \neq 2 \alpha_{q_{1}}-n_{q_{1}}$ then the orbit of $x$ is three-dimensional, and is two-dimensional otherwise.

We shall assume we are in the latter case.

Thus the image of $x$ under the action of an element $A \in \mathrm{SL}_{2}(\mathbb{R})$ is given by the images $t_{1}$ and $t_{2}$ of 0 and $\infty$ under the action of $A$ on $\mathbb{R P}^{1}$. If $\alpha_{q}=n_{q} / 2$ for all $q(x$ is therefore of even support) and $\alpha_{q_{+}}-\alpha_{q}$ is even for all $q$ then exchanging $t_{1}$ and $t_{2}$ gives the same point of the orbit, else it does not.

\section{Closure of low dimensional algebraic orbits}

We shall now determine the closures of the orbits.

By the border of an orbit $O$ we mean the set $\bar{O} \backslash O$.

\subsection{Elliptic case}

We shall consider the orbit of the element $x$ which is elliptic, associated to $\imath$ and $\left[u_{q}\right]_{q}$, that is : $x=\left[u_{q}(\imath X+Y)^{\frac{n_{q}}{2}}(-\imath X+Y)^{\frac{n_{q}}{2}}\right]_{q}$.

Lemma 3.1 The border of the orbit of an elliptic element $x$ associated to a projective point $\left[u_{q}\right]_{q}$ is the circular parabolic orbit of $\left[u_{q} Y^{n_{q}}\right]_{q \in I_{+}(x)}$. The union of these two orbits is a closed disk.

Proof We have

$$
\left(\begin{array}{ll}
a & b \\
c & d
\end{array}\right) \cdot x=\left[u_{q}|c l+d|^{n_{q}-n_{q_{+}}}\left(\frac{a l+b}{c l+d} X+Y\right)^{\frac{n_{q}}{2}}\left(\overline{\frac{a l+b}{c l+d}} X+Y\right)^{\frac{n_{q}}{2}}\right]_{q} .
$$

Since $a d-b c=1$, we can write:

$$
\frac{a \imath+b}{c \imath+d}=\frac{a c+b d}{|c \imath+d|^{2}}+\imath \frac{1}{|c \imath+d|^{2}}
$$

thus $|c l+d|^{2}=(\operatorname{Im} z)^{-1}$, and hence the orbit is the set of the elements

$$
x(z)=\left[u_{q}(\operatorname{Im} z)^{\frac{n_{q_{+}-n_{q}}}{2}}(z X+Y)^{\frac{n_{q}}{2}}(\bar{z} X+Y)^{\frac{n_{q}}{2}}\right]_{q},
$$

where $z \in \mathbb{H}^{+}$.

If a sequence $\left(x\left(z_{i}\right)\right)_{i}$ has a limit in $\mathbb{P}(V)$, necessarily $\left(z_{i}\right)_{i}$ has a limit in the closure of $\mathbb{H}^{+}$in $\overline{\mathbb{C}}$. If this limit is in $\mathbb{H}^{+}$we get a point of the orbit of $x$, otherwise it is a point $t \in \partial \mathbb{H}^{+}$. In the latter case, if $t$ is finite, $\operatorname{Im} z_{i}$ has limit zero and $\left(x\left(z_{i}\right)\right)_{i}$ has limit $\left[u_{q}(t X+Y)^{n_{q}}\right]_{q \in I_{+}(x)}$. If $t=\infty,\left(x\left(z_{i}\right)\right)_{i}$ has limit $\left[u_{q} X^{n_{q}}\right]_{q \in I_{+}(x)}$, which we can write $\left[u_{q}(\infty X+Y)^{n_{q}}\right]_{q \in I_{+}(x)}$. 


\subsection{Parabolic case}

The circular orbits are closed, so we consider only the two types of cylindric orbits; as the technique is the same than in the elliptic case, we shall not give much detail.

Lemma 3.2 Let $x=\left[u_{q} Y^{n_{q}}\right]_{q}$ be of even non-reduced to a single element support. The border of the cylindric orbit of $x$ is the disjoint union of the orbits of $\left[u_{q} Y^{n_{q}}\right]_{q \in I_{+}(x)}$ and $\left[u_{q} Y^{n_{q}}\right]_{q \in I_{-}(x)}$.

If the support of $x$ has a parity (i.e., is even or odd), the closure of the orbit of $x$ is a closed cylinder if $n_{q_{-}}>0$ and a closed disk if $n_{q_{-}}=0$.

If the support of $x$ is neither odd nor even, the closure of the orbit of $x$ is a Klein bottle if $n_{q_{-}}>0$ and a projective plane if $n_{q_{-}}=0$.

Proof We shall consider the orbit of an element $x=\left[u_{q} Y^{n_{q}}\right]_{q}$ whose support is even and has at least two elements. This orbit is described in Sect. 2.5, we can write it under the form:

$$
\begin{aligned}
& \left(\begin{array}{ll}
a & b \\
c & d
\end{array}\right) \cdot x=\left[u_{q} d^{n_{q}-n_{q_{ \pm}}}\left(\frac{b}{d} X+Y\right)^{n_{q}}\right]_{q} \text { if } d \neq 0, \\
& \left(\begin{array}{ll}
a & b \\
c & 0
\end{array}\right) \cdot x=\left[u_{q} b^{n_{q}-n_{q_{ \pm}}} X^{n_{q}}\right]_{q},
\end{aligned}
$$

where we choose \pm to be + (respectively - ) if we want to study great (respectively small) values of the real parameter given for a chosen $t=\frac{b}{d} \in \mathbb{R P}^{1}$ by $d$ (or $b$ if $t=\infty)$.

For great values, we find a point of the circular orbit of $\left[u_{q} Y^{n_{q}}\right]_{q \in I_{+}(x)}$, for small ones a point of the orbit of $\left[u_{q} Y^{n_{q}}\right]_{q \in I_{-}(x)}$ (which is a circle if $n_{q_{-}(x)}>0$, a single point otherwise).

The way the cylindric orbit is glued on the circles of its border depends on the parity of the support of $x$ : if it has a parity (i.e. is even or odd) the couples $(b, d)$ and $(-b,-d)$ of parameters give the same point, else they give two different points such that if one of them is close to a point of the border, the other is close to this point too: hence the cylinder will glue twice on each circle in its border.

\subsection{Hyperbolic case}

Lemma 3.3 The border of the orbit $O$ of an element $x=\left[u_{q} X^{\alpha_{q}} Y^{n_{q}-\alpha_{q}}\right]_{q}$ (where $2 \alpha_{q}-n_{q}$ does not depend upon $\left.q\right)$ is the circular orbit of $\left[u_{q} Y^{n_{q}}\right]_{q \in I_{+}(x)}$.

If $O$ is a Möbius strip, its closure is a closed Möbius strip.

If $O$ is a cylinder, its closure is a torus.

Proof We can write this orbit as the set of all elements of the form

$$
\begin{array}{r}
{\left[u_{q}\left(t_{1}-t_{2}\right)^{\alpha_{q_{+}}-\alpha_{q}}\left(t_{1} X+Y\right)^{\alpha_{q}}\left(t_{2} X+Y\right)^{\beta_{q}}\right]_{q}} \\
=\left[u_{q}\left(\frac{1}{t_{2}}-\frac{1}{t_{1}}\right)^{\alpha_{q_{+}}{ }^{-\alpha_{q}}}\left(X+\frac{1}{t_{1}} Y\right)^{\alpha_{q}}\left(X+\frac{1}{t_{2}} Y\right)^{\beta_{q}}\right]_{q}
\end{array}
$$

with $t_{1}, t_{2} \in \mathbb{R P}^{1}$. As before, this enables the description of the border of this orbit. 


\section{Classification of analytic algebraic action of $S L_{2}(\mathbb{R})$ on surfaces}

We shall now study the analyticity of the different topological surfaces obtained as a union of orbits and which are analytically conjugate (i.e., are equal up to an analytic change of coordinates).

\subsection{Smoothness of polynomial-parametrized surfaces}

We shall use many times the following result, which can be generalized (but we present here only the two-dimensional version for simplicity).

Proposition 4.1 Let $P:\left(x_{1}, x_{2}\right) \longmapsto\left(P_{1}\left(x_{1}, x_{2}\right), \ldots, P_{n}\left(x_{1}, x_{2}\right)\right)$ be a map defined on a neighborhood of 0 in $\mathbb{R}^{2}$ where the $P_{i}$ 's are homogeneous non-constant polynomials. We assume $P_{1}$ to be of minimal degree and $P_{2} \notin \mathbb{R}\left[P_{1}\right]$ of minimal degree among $P_{i}$ 's with that property. If there exists some $P_{i} \notin \mathbb{R}\left[P_{1}, P_{2}\right]$ then the image $E$ of $P$ is not a smooth two-dimensional submanifold of $\mathbb{R}^{n}$ (more precisely, $E$ is singular at 0 ).

Proof Assume that $E$ is a smooth two-dimensional submanifold of $\mathbb{R}^{n}$. Thus there is a smooth implicit definition of $E$, that is to say a neighborhood $U$ of $E$ in $\mathbb{R}^{n}$ and a smooth map $h: U \longrightarrow \mathbb{R}^{n-2}$ of rank $n-2$ everywhere such that $E=\{x \in U ; h(x)=0\}$.

Moreover, assume there is a polynomial $P_{i_{0}} \notin \mathbb{R}\left[P_{1}, P_{2}\right]$ (we choose it of minimal degree).

Let $d$ be the degree of $P_{1}$. We consider the Taylor developpement of order 1 of $h$ in 0 and estimate it in $\left(P_{1}\left(x_{1}, x_{2}\right), \ldots, P_{n}\left(x_{1}, x_{2}\right)\right)$. Noting $h_{j}$ the $j$ th coordinate fonction of $h$ and $\partial_{i}$ the derivation in the $i$ th variable, we get for each $j$ :

$$
0=\sum_{i} \partial_{i} h_{j}(0) P_{i}\left(x_{1}, x_{2}\right)+o\left(\left\|x_{1}, x_{2}\right\|^{d}\right)
$$

where the sum is taken over the $P_{i}$ 's of degree $d$, hence

$$
\left(\begin{array}{ccc}
\partial_{1} h_{1}(0) & \ldots & \partial_{n} h_{1}(0) \\
\vdots & & \vdots \\
\partial_{1} h_{n-2}(0) & \ldots & \partial_{n} h_{n-2}(0)
\end{array}\right)\left(\begin{array}{c}
P_{1} \\
P_{2} \text { if it is of degree } d, 0 \text { otherwise } \\
\vdots \\
P_{i} \text { if it is of degree } d, 0 \text { otherwise } \\
\vdots \\
P_{n} \text { if it is of degree } d, 0 \text { otherwise }
\end{array}\right)=0 .
$$

Each line in the second matrix is given by the coefficients of the corresponding polynomial.

First assume that $P_{2}$ and $P_{i_{0}}$ are both of degree $d$. Thus the family of $P_{i}$ 's of degree $d$ is of rank at least 3 , hence the jacobian matrix of $h$ at the point 0 is of rank at most $n-3$ which prevent $h$ from being an implicit definition of $E$.

Next assume that $P_{2}$ is of degree $d$ and $P_{i_{0}}$ of degree $d_{0}$ greater than $d$. Thus $h$ is of corank at least 2 at the point 0: we have two independent linear combinations of the $\partial_{i} h(0)$ 's which must be zero and involve only the indices $i$ of degree $d$ polynomials. But we can now use the Taylor developpement of order $d_{0}$ to get for each $j$ :

$$
0=\sum_{i} \partial_{i} h_{j}(0) P_{i}\left(x_{1}, x_{2}\right)+Q_{j}\left(x_{1}, x_{2}\right),
$$


where the sum is taken over all polynomials of degree $d_{0}$ which are not in $\mathbb{R}\left[P_{1}, P_{2}\right]$ and $Q_{j}$ is a polynomial of degree $d_{0}$ of $\mathbb{R}\left[P_{1}, P_{2}\right]$. Let $\mathbb{R}^{d_{0}}\left[P_{1}, P_{2}\right]$ be the space of polynomials of $\mathbb{R}\left[P_{1}, P_{2}\right]$ that are homogeneous of degree $d_{0}$. Let $S$ be, in the vector space of all homogenous polynomials of degree $d_{0}$, a supplementary of $\mathbb{R}^{d_{0}}\left[P_{1}, P_{2}\right]$. Let $\tilde{P}_{i}$ be the projection of $P_{i}$ on $S$ along $\mathbb{R}^{d_{0}}\left[P_{1}, P_{2}\right]$. Thus we have for each $j$ :

$$
0=\sum_{i} \partial_{i} h_{j}(0) \tilde{P}_{i}\left(x_{1}, x_{2}\right)
$$

where the sum is taken over all polynomials of degree $d_{0}$ which are not in $\mathbb{R}\left[P_{1}, P_{2}\right]$. As before, it gives a linear combination of the $\partial_{i} h(0)$ 's which must be zero, and is independent of the two we get previously as $P_{i_{0}} \notin \mathbb{R}\left[P_{1}, P_{2}\right]$. Hence $h$ is of corank at least 3 in $O$ and the contradiction holds as before.

We can use the same proof for the case when $P_{2}$ is of degree greater than $d$.

\subsection{Compactifications of the hyperbolic plane: the elliptic case}

\subsubsection{Analytic nonnecessarily algebraic compactification}

We shall start with a description of all analytic compactifications of $\mathcal{E}$ into a closed disk, in the following sense:

Definition 4.2 A differentiable compactification of a differentiable action $\alpha$ of a Lie group $G$ on a manifold $M$ is a triple $(N, \phi, \bar{\alpha})$ where $N$ is a compact manifold with boundary, $\bar{\alpha}$ is a differentiable action of $G$ on $N, \phi: M, \alpha \longrightarrow N, \bar{\alpha}$ is an equivariant embedding such that $\phi(M)$ is dense in $N$.

The work of Schneider [2], Stowe [4] exposed by Mitsumatsu [1] gives the classification of all such compactifications, which we recall in what follows.

We shall use the following basis for $\mathfrak{s l}_{2}(\mathbb{R})$ :

$$
H=\left(\begin{array}{cc}
1 & 0 \\
0 & -1
\end{array}\right), \quad K=\left(\begin{array}{cc}
0 & -1 \\
1 & 0
\end{array}\right), \quad L=\left(\begin{array}{ll}
0 & 1 \\
1 & 0
\end{array}\right) .
$$

The infinitesimal generators for the projective compactification are given on $\mathbb{R} \times \mathbb{R}_{+}$by

$$
\begin{aligned}
& \bar{K}_{1+}=2 \frac{\partial}{\partial x}, \\
& \bar{H}_{1+}=2\left((\sin x)(1+y) \frac{\partial}{\partial x}+(\cos x)\left(2 y+y^{2}\right) \frac{\partial}{\partial y}\right), \\
& \bar{L}_{1+}=2\left((\cos x)(1+y) \frac{\partial}{\partial x}-(\sin x)\left(2 y+y^{2}\right) \frac{\partial}{\partial y}\right) .
\end{aligned}
$$

and can be completed by adding a point at infinity.

Theorem $4.3([1,2,4])$ By pulling back the restriction of the vector fields $\bar{K}_{1+}, \bar{H}_{1+}$, $\bar{L}_{1+}$ to $\mathbb{R} \times \mathbb{R}_{+}^{*}$ by the map $F_{n}(x, y)=\left(x, y^{n}\right)$ where $n$ is a nonnegative integer and by taking their continuous prolongations, we get analytic vector fields $\bar{K}_{n+}, \bar{H}_{n+}, \bar{L}_{n+}$ on $\mathbb{R} \times \mathbb{R}_{+}$. For any analytic compactifications of $\mathcal{E}$ into a closed disk, there is an unique $n$ and $a \mathbb{R} \times \mathbb{R}_{+}$chart in which these vector fields are the infinitesimal generators of the compactified action. 
For example, $\bar{K}_{2+}, \bar{H}_{2+}, \bar{L}_{2+}$ are the infinitesimal generators for the conformal compactification.

\subsubsection{Analytic algebraic compactifications}

We shall now study the algebraic analytic compactifications of $\mathcal{E}$ into a closed disk, that is to say the elliptic orbits whose closure is an analytic submanifold with boundary in the projective space $\mathbb{P}(V)$.

We prove a more precise version of the theorem 1.3 exposed in the introduction:

Theorem 4.4 Let $O$ be the orbit of

$$
x=\left[u_{q}(\imath X+Y)^{\frac{n_{q}}{2}}(-\imath X+Y)^{\frac{n_{q}}{2}}\right]_{q} .
$$

If all the element of the family $\left(\left(n_{q_{+}}-n_{q}\right) / 2\right)_{q \in I(x)}$ are even, then $\bar{O}$ is an analytic submanifold with boundary and the action of $\mathrm{SL}_{2}(\mathbb{R})$ on this disk is conjugate to the projective action.

If there exists some $q_{2+}$ in $I(x)$ such that $\left(\left(n_{q_{+}}-n_{q_{2+}}\right) / 2\right)=1$, then $\bar{O}$ is an analytic submanifold with boundary and the action of $\mathrm{SL}_{2}(\mathbb{R})$ on this disk is conjugate to the conformal action.

In all the other cases, $\bar{O}$ is not an analytic submanifold with boundary.

Proof The methods used here will be useful through all the following sections.

We shall first consider the case when all the numbers $\left(n_{q_{+}}-n_{q}\right) / 2$, where $q$ is in $I(x)$, are even. A model for the projective compactification is given by the closure in $\mathbb{P}\left(\mathbb{R}_{2}[X, Y]\right)$ of the orbit of $\left[X^{2}+Y^{2}\right]$, which is contained in the affine chart $\left\{\left[a X^{2}+b X Y+(1-a) Y^{2}\right] ; a, b \in \mathbb{R}\right\}$. The map

$$
\begin{aligned}
\varphi: \mathbb{P}\left(\mathbb{R}_{2}[X, Y]\right) \longrightarrow & \mathbb{P}(V)\left[a X^{2}+b X Y+(1-a) Y^{2}\right] \\
\longmapsto & {\left[\begin{array}{l}
u_{q}\left(a(1-a)-\frac{b^{2}}{4}\right)^{\frac{n_{q_{+}-n_{q}}}{4}} \\
\left.\times\left(a X^{2}+b X Y+(1-a) Y^{2}\right)^{\frac{n_{q}}{2}}\right]_{q}
\end{array}\right.}
\end{aligned}
$$

is injective, analytic (thanks to the hypothesis) and realizes a conjugacy between the projective action and the dynamics on $\bar{O}$.

Moreover, it is an immersion since, noting $s, t, u, v$ the coefficients of the terms in $X^{n_{q_{+}}}, X^{n_{q_{+}-1}} Y, Y^{n_{q_{+}}}, X Y^{n_{q_{+}-1}}$, we have

$$
\begin{aligned}
& \frac{\partial s}{\partial a}=\frac{\partial t}{\partial b}=\frac{n_{q_{+}}}{2} a^{\frac{n_{q_{+}}}{2}-1} \\
& \frac{\partial u}{\partial a}=-\frac{\partial v}{\partial b}=-\frac{n_{q_{+}}}{2}(1-a)^{\frac{n_{q_{+}}}{2}-1} \\
& \frac{\partial s}{\partial b}=\frac{\partial u}{\partial b}=0
\end{aligned}
$$

hence the differential of $\varphi$ is of rank 2 everywhere. 
This proves that $\bar{O}$ is an analytic submanifold with boundary and that the action of $\mathrm{SL}_{2}(\mathbb{R})$ on it is conjugate to the projective one.

Next we shall consider the case when there exists some $q_{2+}$ in $I(x)$ such that $\left(n_{q_{+}}-n_{q_{2+}}\right) / 2=1$. A model for the conformal action is given by the closure of $\mathbb{H}^{+}$in the Riemann sphere. We consider the map

$$
\begin{aligned}
\psi: \overline{\mathbb{H}^{+}} & \longrightarrow \mathbb{P}(V) \\
a+\imath b & \longmapsto\left[u_{q} b^{\frac{n_{q_{+}-n_{q}}}{2}}((a+\imath b) X+Y)^{\frac{n_{q}}{2}}((a-\imath b) X+Y)^{\frac{n_{q}}{2}}\right]_{q}
\end{aligned}
$$

which is injective, analytic and realizes a conjugacy between the conformal action and the dynamics on $\bar{O}$. Notice that $\psi(\infty)=\left[u_{q} X^{n_{q}}\right]_{q \in I_{+}(x)}$.

Moreover, developing the expression of $\psi(a+\imath b)$, we see that a coefficient is $n_{q_{+}} a$ and another is $u_{q_{2+}} b$, so $\psi$ is everywhere of rank 2 and we can conclude as before.

For the last case, we use Proposition 4.1. We denote by $\alpha$ the smallest odd element of the family $\left(\left(n_{q_{+}}-n_{q}\right) / 2\right)_{q}$, we denote by $q_{2+}$ an index realizing this minimum. By hypothesis $\alpha>1$. We can write an element of $\bar{O}$ under the form:

$$
\left[u_{q}(\operatorname{Im} z)^{\frac{n_{q_{+}-n_{q}}}{2}}\left(\left(\operatorname{Im} z^{2}+\operatorname{Re} z^{2}\right) X^{2}+2 \operatorname{Re} z X Y+Y^{2}\right)^{\frac{n_{q}}{2}}\right]_{q} .
$$

All coordinates are homogeneous polynomials in $x=\operatorname{Re} z$ and $y=\operatorname{Im} z$. Among them $P_{1}=x$ (we define it up to a multiplicative constant) is of minimal degree. Among those which are not in $\mathbb{R}\left[P_{1}\right], P_{2}=y^{2}$ is of minimal degree. But $P_{3}=y^{\alpha} \notin \mathbb{R}\left[P_{1}, P_{2}\right]$ hence $\bar{O}$ is not a smooth submanifold of $\mathbb{P}(\mathrm{V})$, therefore not an analytic one.

Remark 4.5 In this proof we can see more than stated: the embeddings $\varphi$ and $\psi$ extend respectively to embeddings of a projective plane and a sphere.

The projective plane is the union of the elliptic orbit of $x$, the hyperbolic orbit of

$$
\left[\left(-\frac{1}{4}\right)^{\frac{n_{q_{+}-n_{q}}}{4}} u_{q} X^{\frac{n_{q}}{2}} Y^{\frac{n_{q}}{2}}\right]_{q}
$$

which is a Möbius strip and their common border, namely the circular orbit of $\left[u_{q} Y^{n_{q}}\right]_{q \in I_{+}(x)}$.

The sphere is the union of the elliptic orbits of $x$ and of

$$
\left[(-1)^{\frac{n_{q_{+}-n_{q}}}{2}} u_{q}\left(-X^{2}+Y^{2}\right)^{\frac{n_{q}}{2}}\right]_{q}
$$

and of their common border, the circular orbit of $\left[u_{q} Y^{n_{q}}\right]_{q \in I_{+}(x)}$.

Moreover, we see that if we are in the third case, the map $\varphi$ is not analytic but is a $\mathcal{C}^{\frac{\alpha-1}{2}}$ embedding of the projective action, so we can state the following fact concerning the differentiable case for elliptic orbits:

Theorem 4.6 The only algebraic differentiable compactifications of $\mathcal{E}$ are equivalent to the projective or to the conformal ones. In the projective case there exist $\mathcal{C}^{k}$ non-analytic realizations for each finite $k$, but any $\mathcal{C}^{\infty}$ realization is in fact analytic. In the conformal case any $\mathcal{C}^{1}$ realization is in fact analytic. 
4.3 Hyperbolic case

Here we shall consider the closure of a hyperbolic two-dimensional orbit, which has the form

$$
\bar{O}=\left\{\left[u_{q}\left(t_{1}-t_{2}\right)^{\alpha_{q_{+}}-\alpha_{q}}\left(t_{1} X+Y\right)^{\alpha_{q}}\left(t_{2} X+Y\right)^{n_{q}-\alpha_{q}}\right]_{q} ; t_{1}, t_{2} \in \mathbb{R P}^{1}\right\} .
$$

Theorem 4.7 If $O$ is a Möbius strip (i.e., for each $q, n_{q}$ is even, $\alpha_{q}=\frac{n_{q}}{2}$ and $\alpha_{q_{+}}-\alpha_{q}$ is even), $\bar{O}$ is an analytic submanifold; moreover its union with the elliptic orbit of

$$
\left[\left(-\frac{1}{4}\right)^{\frac{n_{q_{+}-n_{q}}}{4}} u_{q}\left(X^{n_{q}}+Y^{n_{q}}\right)\right]
$$

is still analytic and the dynamics is conjugate to the projective action of $\mathrm{SL}_{2}(\mathbb{R})$ on the projective plane.

If there is some $q_{2+}$ such that $\alpha_{q_{+}}-\alpha_{q_{2+}}=1, \bar{O}$ is an analytic submanifold of $\mathbb{P}(V)$ and its dynamics is conjugate to the natural product action of $\mathrm{SL}_{2}(\mathbb{R})$ on $\mathbb{R P} \mathbb{P}^{1} \times \mathbb{R} \mathbb{P}^{1}$.

In all the other cases, $\bar{O}$ is not an analytic submanifold.

Proof The first case is given by the map $\varphi$ of the previous section (see Remark 4.5).

In the second case, we consider the map

$$
\begin{aligned}
\psi: \mathbb{R P}^{1} \times \mathbb{R P}^{1} & \longrightarrow \mathbb{P}(V) \\
\left(t_{1}, t_{2}\right) & \longmapsto\left[u_{q}\left(t_{1}-t_{2}\right)^{\alpha_{q_{+}}-\alpha_{q}}\left(t_{1} X+Y\right)^{\alpha_{q}}\left(t_{2} X+Y\right)^{n_{q}-\alpha_{q}}\right]_{q}
\end{aligned}
$$

which is analytic, injective as the orbit is by hypothesis a cylinder and is an immersion as the coefficient of the terms in $X Y^{n_{q_{+}}}$and $Y^{n_{q_{2}}}$ of $\psi\left(t_{1}, t_{2}\right)$ are respectively $\alpha_{q_{+}} t_{1}+\left(n_{q}-\alpha_{q_{+}}\right) t_{2}$ and $t_{1}-t_{2}$, which gives a partial jacobian matrix $\left(\begin{array}{cc}\alpha_{q_{+}} n_{q}-\alpha_{q_{+}} \\ 1\end{array}\right)$ whose determinant is $-n_{q_{+}} \neq 0$. Hence $\bar{O}$ is an analytic submanifold (without boundary) of $\mathbb{P}(V)$ and (see the topological study) its dynamics is conjugate to the product action of $\mathrm{SL}_{2}(\mathbb{R})$ on $\mathbb{R} \mathbb{P}^{1} \times \mathbb{R P}^{1}$.

For the last case we use Proposition 4.1. The only polynomial of degree 1 among the coordinates is $P_{1}=\alpha t_{1}+\beta t_{2}$ where we write $\alpha$ for $\alpha_{q_{+}}$and $\beta$ for $n_{q_{+}}-\alpha_{q_{+}}$. We can next choose

$$
P_{2}=\frac{\alpha(\alpha-1)}{2} t_{1}^{2}+\alpha \beta t_{1} t_{2}+\frac{\beta(\beta-1)}{2} t_{2}^{2} .
$$

Setting $\tilde{P}_{2}=\left(t_{1}-t_{2}\right)^{2}$, an easy computation gives $\mathbb{R}\left[P_{1}, P_{2}\right]=\mathbb{R}\left[P_{1}, \tilde{P}_{2}\right]$.

If $\alpha=\beta$, as $\bar{O}$ is assumed to be a cylinder there must exist some index $q_{0}$ such that $\alpha_{q_{+}}-\alpha_{q_{0}}$ is odd. Thus one of the coordinates has the form $\left(t_{1}-t_{2}\right)^{\alpha_{q_{+}}-\alpha_{q_{0}} \text { which }}$ is not in $\mathbb{R}\left[P_{1}, P_{2}^{\prime}\right]$, hence from Proposition 4.1 we conclude that $\bar{O}$ is not an analytic submanifold of $\mathbb{P}(V)$.

If $\alpha \neq \beta$, we see after an easy computation that the coordinate

$$
P_{3}=\frac{\alpha(\alpha-1)(\alpha-2)}{6} t_{1}^{3}+\frac{\alpha(\alpha-1)}{2} \beta t_{1}^{2} t_{2}+\alpha \frac{\beta(\beta-1)}{2} t_{1} t_{2}^{2}+\frac{\beta(\beta-1)(\beta-2)}{6} t_{2}^{3}
$$

of the term $X^{3} Y^{n_{q}-3}$ is not in $\mathbb{R}\left[P_{1}, P_{2}\right]$ and the conclusion still holds. 


\subsection{Parabolic case}

We shall finally consider the closure of a parabolic orbit, which has the form

$$
\bar{O}=\left\{\left[u_{q} d^{n_{q}-n_{q_{-}}}(t X+Y)^{n_{q}}\right]_{q} ; d \in \overline{\mathbb{R}} \text { and } t \in \mathbb{R P}^{1}\right\},
$$

where $d \in \overline{\mathbb{R}}$ means $d$ is real or $\pm \infty$.

We shall prove some lemmas before stating the general result. Let $q_{2-}$ (respectively $q_{2+}$ ) be an index such that $n_{q_{2-}}$ (respectively $q_{2+}$ ) is minimal (respectively maximal) among $n_{q}$ 's greater than $n_{q_{-}}$(respectively lesser than $n_{q_{+}}$).

Lemma 4.8 If $n_{q_{-}}=0$ and $\bar{O}$ is a smooth submanifold of $\mathbb{P}(V)$, we must have $n_{q_{2}-}=1$ and hence $\bar{O}$ is a projective plane.

Proof We shall use Proposition 4.1 once again, around the point $\left[u_{q}\right]_{q \in I_{-}}$corresponding to $d=0, t=0$. The least-dimensional non-constant polynomial among the local coordinates is $P_{1}=d^{n_{q_{2}}}$. There is no other polynomial of the same degree, so we can choose $P_{2}=t P_{1} \notin \mathbb{R}\left[P_{1}\right]$. If $n_{q_{2}-}>1$, one of the coordinates can be written as $t^{2} P_{1} \notin \mathbb{R}\left[P_{1}, P_{2}\right]$ and $\bar{O}$ can not be a smooth submanifold of $\mathbb{P}(V)$.

Lemma 4.9 If $\bar{O}$ is a smooth submanifold of $\mathbb{P}(V)$, we must have

- $n_{q_{+}}-n_{q_{2+}}=n_{q_{2-}}-n_{q_{-}}$,

- for each $q, n_{q_{+}}-n_{q_{2+}}$ divides $n_{q_{+}}-n_{q}$.

Proof We use Proposition 4.1 twice.

We first look around the point $\left[u_{q} Y^{n_{q}}\right]_{q \in I_{-}}$to prove that for each $q, n_{q_{2-}}-n_{q_{-}}$ divides $n_{q}-n_{q_{-}}$. If $n_{q_{-}}=0$, we have $n_{q_{2-}}=1$ and the claim is obvious. If $n_{q_{-}}>0$, we can choose $P_{1}=t$ and $P_{2}=d^{n_{q_{2-}}-n_{q_{-}}}$. For each $q$ there is a coordinate which has the form $d^{n_{q}-n_{q_{-}}}$, hence by Proposition $4.1 n_{q_{2-}}-n_{q_{-}}$must divide $n_{q}-n_{q_{-}}$.

In particular $n_{q_{2-}}-n_{q_{-}}$divides $n_{q_{+}}-n_{q_{2+}}$.

We now look around the point $\left[u_{q} Y^{n_{q}}\right]_{q \in I_{+}}$, where local coordinates are given by writing a point of $\bar{O}$ under the form $\left[u_{q} f^{n_{q_{+}}-n_{q}}(t X+Y)^{n_{q}}\right]_{q}$ after a change of coordinates $f=d^{-1}$. We can choose $P_{1}=t$ and $P_{2}=f^{n_{q_{+}}-n_{q_{2+}}}$, thus as there is coordinates of the form $f^{n_{q_{+}}-n_{q}}$, for all $q, n_{q_{+}}-n_{q_{2+}}$ divides $n_{q_{+}}-n_{q}$.

In particular $n_{q_{+}}-n_{q_{2+}}$ divides $n_{q_{2-}}-n_{q_{-}}$and the conclusion holds.

It is easy to see that the necessary conditions given in the previous lemma are also sufficient if $n_{q_{-}} \neq 0$ for $\bar{O}$ to be an analytic submanifold of $\mathbb{P}(V)$ : around each point of $\bar{O}$ we can find local coordinates of the form $P_{k, l}=d^{k\left(n_{q_{+}}-n_{q_{2+}}\right)} t^{l}$ where $k$ and $l$ are integers and for some coordinates we have $(k, l)=(0,1)$ or $(k, l)=(1,0)$, hence writing $P_{k, l}-P_{1,0}{ }^{k} P_{0,1}{ }^{l}=0$ we get an analytic implicit local definition of $\bar{O}$. If $n_{q_{-}}=0$ the combination of the conditions of the two lemmas are also sufficient for $\bar{O}$ to be analytic since we can find local coordinates of the previous form or, around the points given by $d=0$, of the form $P_{k, l}=d^{k} t^{l}$ with $k>0, k \geq l$; for some coordinates we have $(k, l)=(1,1)$ and $(k, l)=(1,0)$ hence we get an analytic implicit local definition of the form $P_{k, l}-P_{1,1}{ }^{l} P_{1,0}{ }^{k-l}=0$. 
Moreover, if we map a point given by parameters $d, t$ from the closure of an analytic parabolic orbit to the point given by the same parameters on another such orbit closure of the same topology (projective plane, Klein bottle or cylinder) and with the same value for $n_{q_{2-}}-n_{q_{-}}$we build an analytic diffeomorphism between them:

$$
\left[u_{q} d^{n_{q}-n_{q_{-}}}(t X+Y)^{n_{q}}\right]_{q} \longmapsto\left(d^{n_{q_{2-}}-n_{q_{-}}}, t\right) \longmapsto\left[u_{q}^{\prime} d^{n_{q}-n_{q_{-}^{\prime}}}(t X+Y)^{n_{q}}\right]_{q} .
$$

Finally, if we consider the differential in the point $x=\left[u_{q} Y^{n_{q_{-}}}\right]_{q \in I_{-}}$of an element $\left(\begin{array}{ll}a & 0 \\ c & a^{-1}\end{array}\right)$ of the stabilizer of $x$ we find that its eigenvalues are $a^{-2}$ and $a^{-\left(n_{q_{2-}}{ }^{\left.-n_{q_{-}}\right)} \text {, so }\right.}$ two closures of orbits with different values of $n_{q_{2-}}-n_{q_{-}}$can not be differentiably conjugate. Hence we can state:

Theorem 4.10 The conditions of Lemmas 4.8 and 4.9 are sufficient for $\bar{O}$ to be an analytic submanifold of $\mathbb{P}(V)$. Two analytic parabolic orbits are analyticaly conjugate if and only if they have the same topology and the same value for $n_{q_{2-}}-n_{q_{-}}$(and they are not even differentiably conjugate otherwise). In particular there is one parabolic algebraic action on the projective plane, a countable family of actions on the Klein bottle and a countable family of actions on the closed cylinder.

The last point we have to study in order to complete the proof of the results stated in the introduction is the way the cylindric orbits are glued together.

Let $O$ be a cylindric analytic orbit associated to a projective element $\left[u_{q}\right]_{q}$. Its boundary is the union of the two circular orbits associated to the projective elements $\left[u_{q}\right]_{q \in I_{+}}$and $\left[u_{q}\right]_{q \in I_{-}}$, which we call respectively the upper component and the lower component of the boundary.

An element of $\bar{O}$ can be written $\left[u_{q} d^{n_{q}-n_{q_{-}}}(t X+Y)^{n_{q}}\right]_{q}$ around the lower component of the boundary. For each $q$ we denote by $k_{q}$ the integer $\left(n_{q}-n_{q_{-}}\right) /\left(n_{q_{2_{-}}}-n_{q_{-}}\right)$. The coordinates $c_{q, l}=u_{q} d^{n_{q}-n_{q_{-}}} t^{l}$ satisfy the implicit definition given previously:

$$
\frac{1}{u_{q}} c_{q, l}-\frac{1}{u_{q_{2-}}} c_{q_{2-}, 0} k_{q} \frac{1}{u_{q_{-}} n_{q_{-}}} c_{q_{-}, 1}{ }^{l}=0 .
$$

Let $O^{\prime}$ be the cylindric analytic orbit associated with the projective element $\left[u_{q}^{\prime}\right]_{q}$ where $u_{q}^{\prime}=(-1)^{k_{q}} u_{q}$. Thus the lower component of its boundary is the same than for $O$ and as around it the coordinates of $O^{\prime}$ satisfy the same implicit parametrization, $O$ and $O^{\prime}$ are analytically glued together around their lower component.

With the same method we see that $O$ and the orbit $O^{\prime \prime}$ associated with $\left[u_{q}^{\prime \prime}\right]_{q}$ where $u_{q}^{\prime \prime}=(-1)^{k_{q_{+}}-k_{q}} u_{q}$ are analytically glued around their common upper component.

If $k_{q_{+}}$is even $O^{\prime}=O^{\prime \prime}$ and $O$ together with $O^{\prime}$ gives a torus with two open orbits, if $k_{q_{+}}$is odd $O^{\prime} \neq O^{\prime \prime}$ but they are both glued analytically with $O^{\prime \prime \prime}$, the parabolic orbit associated with $\left[(-1)^{k_{q+}} u_{q}\right]_{q}$. Hence we have proven the last remaining result:

Theorem 4.11 Let $O$ be a parabolic, cylindric, analytic orbit associated to $\left[u_{q}\right]_{q}$.

If $k_{q_{+}}=\left(n_{q_{+}}-n_{q_{-}}\right) /\left(n_{q_{2-}}-n_{q_{-}}\right)$is even, the closure of the union of the two parabolic orbits associated to $\left[u_{q}\right]_{q}$ and $\left[(-1)^{k_{q}} u_{q}\right]_{q}$ is a torus analytically embedded in $\mathbb{P}(V)$.

If $k_{q_{+}}$is odd, the closure of the union of the four parabolic orbits associated to $\left[u_{q}\right]_{q},\left[(-1)^{k_{q}} u_{q}\right]_{q},\left[(-1)^{k_{q_{+}}-k_{q}} u_{q}\right]_{q}$ and $\left[(-1)^{k_{q_{+}}} u_{q}\right]_{q}$ is a torus analytically embedded in $\mathbb{P}(V)$. 


\subsection{Proof of Theorem 1.4}

We gather the preceding results to prove Theorem 1.4.

From Lemma 2.4 we know that we can restrict ourselves to the three cases: elliptic ( $k=0$ and $l=1)$, parabolic $(k=1$ and $l=0)$ and hyperbolic $(k=2$ and $l=0)$. Lemmas 2.5, 2.6 and 2.7 give caracterisations of low-dimensional orbits and describe their topology: elliptic orbits are disks, parabolic orbits are circles or cylinders, hyperbolic orbits are cylinders, Möbius strips or three-dimensional.

In Sect. 3 we determined the topology of the closures of the two-dimensional orbits. The border of an elliptic orbit is a parabolic circle and together they form a closed disk. The border of a parabolic cylinder is the union of two parabolic circles or of a parabolic circle and a point. Depending how the cylinder is glued to its boundary, its closure is a closed disk, a closed cylinder, a Klein bottle or a projective plane. The border of a two-dimensional hyperbolic orbit is a single parabolic circle and together they form a closed Möbius strip or a torus.

With Theorem 4.4 and Remark 4.5 we get the algebraicity of the projective action on the projective plane and of the conformal action on the sphere.

With Theorem 4.7 we saw that a hyperbolic closed Möbius strip is always analytic, and is part of a projective plane with projective action. Moreover, some hyperbolic torus are not analytic, the other are all conjugate with the usual product action of $\mathrm{SL}_{2}(\mathbb{R})$ on $\mathbb{R} \mathbb{P}^{1} \times \mathbb{R} \mathbb{P}^{1}$.

At this point, we got three different algebraic actions of our classification (the projective one, the conformal one and the product torus). The other ones come from gluing of parabolic orbits.

With Theorem 4.10 we saw that the parabolic projective disks give exactly one algebraic action on the projective plane - which has an open (parabolic) orbit - a countable family of algebraic actions on the Klein bottle and a countable family of algebraic actions on the cylinder. Theorem 4.11 shows that two or four of these parabolic cylinders can glue together to form two countable families of algebraic actions on the torus. The classification is complete.

\section{References}

1. Mitsumatsu, Y.: SL(2;R)-actions on surfaces. In Geometric study of foliations (Tokyo, 1993), pp. 375-389. World Sci. Publishing, River Edge, NJ (1994)

2. Schneider, C.R.: SL $(2, R)$ actions on surfaces. Amer. J. Math. 96, 511-528 (1974)

3. Serre, J.-P.: Algèbres de Lie semi-simples complexes.511-528 W. A. Benjamin, New York (1966)

4. Stowe, D.C.: Real analytic actions of $\operatorname{SL}(2, \mathbf{R})$ on a surface. Ergodic Theory Dynam. Syst. 3(3), 447-499 (1983) 